\title{
Neuropsychotherapie: Eine Frage der Verantwortung?
}

\author{
Dirk Hellhammer \\ FB I - Psychobiologie, Universität Trier, Deutschland
}

Mit seinem 2004 veröffentlichten Buch «Neuropsychotherapie» gelang es Klaus Grawe, eine breite Diskussion um Nutzen und Brauchbarkeit neurowissenschaftlicher Erkenntnisse für die Psychotherapieforschung anzustoßen. Derartige Überlegungen haben zwar eine über hundertjährige Tradition, sie wurden aber erst durch dieses Buch, seinen prominenten Autor und durch den griffigen Titel wirklich populär. Grawe war überzeugt, dass der Verhaltenstherapie nach der kognitiven Wende nun auch eine neurowissenschaftliche Wende bevorsteht. Er war der Auffassung, dass jedem Bewusstseinsprozess und somit auch jeder Verhaltensstörung letztendlich beschreibbare neuronale Ereignisse zugrunde liegen. Entsprechend folgerte er, dass sich nicht nur mit Pharmakotherapie, sondern auch durch Psychotherapie neuronale Funktionen ändern lassen. Dazu sei die Verhaltenstherapie in besonderem Maße prädestiniert. Mit seinem Buch unternahm er den Versuch aufzuzeigen, wie sich neurowissenschaftliches Wissen auf die Therapieplanung und Therapiedurchführung auswirken kann.

Klaus Grawe stimmte freudig zu, als ich ihn im vergangenen Jahr im Auftrag der Hauptschriftleitung von VERHALTENSTHERAPIE fragte, ob er Interesse hätte, ein Sonderheft unserer Zeitschrift zum Thema «Neuropsychotherapie» herauszugeben. Nachdem er uns kurz darauf für immer verlassen hat, war es an mir, diese Aufgabe zu übernehmen. Das vor uns liegende Heft kam so rasch zustande, weil alle angesprochenen Au- toren und Autorinnen sofort einem Beitrag zustimmten, wohl auch um Klaus Grawe auf diese Weise posthum Referenz zu erweisen. Die einzelnen Arbeiten zeigen ein breites Spektrum von experimentellen bis zu therapeutischen Ansätzen, mit unterschiedlichen Alters- und Patientengruppen.

Albert [1975] hat vor mehr als 30 Jahren geschrieben, dass ein Psychotherapeut seiner Verantwortung gegenüber dem $\mathrm{Pa}-$ tienten nur gerecht wird, wenn er alle hilfreichen Erkenntnisse, insbesondere die neurowissenschaftlichen, in seine Behandlung einbezieht. So kann man mit Niels Birbaumer (siehe das Interview in diesem Heft) sagen, dass das Ausmaß des Fortschritts stets vom Engagement des einzelnen Wissenschaftlers oder Therapeuten abhängt. Das kann nicht nur den Universitäten und Weiterbildungseinrichtungen überlassen bleiben, die sich dieser Entwicklung stellen müssen, sondern wird auch von dem Verantwortungsbewusstsein und Engagement jedes einzelnen Psychotherapeuten abhängen. Es bleibt daher abzuwarten, ob und wann die Verhaltenstherapie eine neurowissenschaftliche Wende durchläuft.

\section{Literatur}

Albert G: Need therapists be neuropsychologists, too? J Contemp Psychother 1975;7:103-108.

Grawe K: Neuropsychotherapie. Göttingen, Hogrefe, 2004.

\begin{tabular}{ll}
\hline KARGER & @ 2006 S. Karger GmbH, Freiburg \\
Fax +497614520714 & Accessible online at: \\
$\begin{array}{l}\text { E-mail Information@Karger.de } \\
\text { www.karger.com }\end{array}$ & www.karger.com/ver
\end{tabular}

